\title{
Effect of pulse vaccination on dynamics of dengue with periodic transmission functions
}

Rashid Jan ${ }^{1}$ and Yanni Xiao ${ }^{1 *}$

"Correspondence:

yxiao@mail.xjtu.edu.cn

'School of Mathematics and

Statistics, Xi'an Jiaotong University,

Xi'an, P.R. China

\section{Springer}

\begin{abstract}
Pulse vaccination is a repeated vaccination policy, which plays a tremendous role in the global fight against communicable diseases in terms of saving medical resources and decreasing the economic burden. In this article, we propose a dynamic model of dengue infection with periodic transmission functions and seasonality in vector population. Furthermore, we introduce a pulse vaccination strategy in the susceptible host population to examine how frequency and intensity of implementation of this strategy affect the dynamics of dengue infection. We successfully obtained the threshold dynamics by defining the basic reproduction number $R_{0}$, which is the spectral radius of the next generation operator and governs whether the disease dies out or not. It has been established that the infection-free periodic solution of the proposed impulsive system is globally asymptotically stable if $R_{0}<1$ and is unstable otherwise. Moreover, we found that the dengue infection is uniformly persistent for the proposed system if $R_{0}>1$. Finally, we execute the system numerically to illustrate the piecewise solutions of the proposed system with impulsive vaccination measure and to investigate the influence of different control parameters on the basic reproduction. The finding indicates that a frequent implementation of the vaccination strategy with great intensity and the use of mosquito nets can essentially lead to a decline of new infections.
\end{abstract}

Keywords: Dengue fever; Periodic transmission; Impulsive vaccination; Asymptotical stability; Uniform persistence; Numerical simulations

\section{Introduction}

It is evident that vector-borne diseases are induced by pathogenic agents such as helminths, viruses, protozoa, and bacteria. They are transmitted by arthropod vectors, which include bedbugs, biting midges, fleas, lice, kissing bugs, mosquitoes, ticks, sand flies, mites and black flies. It is a fact that dengue infection spreads more rapidly than other vectorborne infections, which causes significant health, economic and social burdens [1, 2]. The symptoms of this viral infection are high fever, red eyes, joints pain, muscles pain, lower back pain, pain behind eyes, nausea, and vomiting, a red flush to the face, severe fatigue, severe weakness and even death [3-8]. The Aedes mosquitoes transfer four different serotypes to the host through their bites when they suck the blood of the host. Humans may be infected with dengue virus more than once because it is caused by four different viruses which are carried by female Aedes mosquitoes [9]. Dengue fever is rarely fatal, but

(c) The Author(s) 2019. This article is distributed under the terms of the Creative Commons Attribution 4.0 International License (http://creativecommons.org/licenses/by/4.0/), which permits unrestricted use, distribution, and reproduction in any medium, provided you give appropriate credit to the original author(s) and the source, provide a link to the Creative Commons license, and indicate if changes were made. 
more severe forms of dengue infections are DSS and DHF, causing 22,000 deaths annually [10]. Moreover, dengue virus is recognized as the major arbovirus on the globe, and each year produces more than 50 million infections $[11,12]$.

It is evident that vaccination plays an essential part in eliminating infectious diseases and is used as an effective method to eliminate the infection by reducing the strength of the susceptible group in the community [13-21]. The impact of impulsive vaccination on an epidemic model with regular constant vaccination was studied in [20], where a fraction of the susceptible class was partially transferred to the vaccination class. In [15], the author explored and conceptualized the role of vaccination in the epidemic model and further highlighted the influence of vaccination on the infection. Even though vaccination is the most powerful tool to eradicate and reduce the level of infection, however, most types of it are not fully effective and do not provide complete immunity to the infection. As a result, mathematical models with vaccination strategy provide vital information to decision makers at both global and national levels. Although several epidemic models have been introduced in previous research to conceptualize and explore the dynamics of dengue infection to detect the leading factors that significantly affect the transmission of the infection [22-32], few have focused on modeling the effect of vaccination on dengue dynamics and this falls within the scope of this research.

A common assumption for the infectious model with vaccination strategy is that the immunization activities happen continuously. However, this is not how things look like. Generally, the immunization happens in regular intervals, and consequently, the continuous human's intervention measure is then removed from the system, and replaced by an impulsive perturbation. Thus, mathematical models with impulsive vaccination have been proposed and investigated [33-40]. In [38,39], the authors inspected the delay epidemic models with the influence of impulsive vaccination, moreover, the stability of the linear impulsive system was investigated in [41]. Note that individuals, who have immunization to one dengue virus due to infection or vaccination, can also be infected by another dengue virus. So the interesting problem is how to design the vaccination regime (i.e., as regards the vaccination intensity and how frequently the vaccination should be implemented) to effectively control the dengue infection.

The target of this work is to initiate a dynamical model for dengue fever with periodic transmission functions and pulse vaccination. The preeminent motivation behind this research is to examine the level of disease after implementation of impulsive vaccination and to investigate how the impulsive policy influences the dynamics of dengue infection. This article is ordered as follows: in Sect. 2 of the article, we introduce the dynamics of dengue fever with periodic transmission functions and impulsive vaccination strategies. In Sect. 3, we reveal that the infection-free periodic solution is GAS for $R_{0}$ less than 1 and is unstable in other respects. Furthermore, the uniform persistence of dengue infection has been shown in the proposed impulsive system for $R_{0}>1$. In Sect. 4 , we carry out some numerical simulation to elucidate the impulsive system to inspect the feasibility of the impulsive vaccination control strategy. Finally, we present endemic indicator in terms of different parameters of the system numerically.

\section{Formulation of the model}

To formulate the interaction of vector and human for dengue infection, we indicate the total female vector size by $N_{v}$ and the total human size by $N_{h}$. The total female 
vector size is categorized into susceptible $\left(S_{v}\right)$ and infectious $\left(I_{v}\right)$ states while the human population $N_{h}$ is categorized into susceptible human $\left(S_{h_{1}}\right)$, susceptible human with partial immunity $\left(S_{h_{2}}\right)$, infected $\left(I_{h}\right)$ and recovered $\left(R_{h}\right)$ states. Moreover, it is assumed that negligible mortality is produced by dengue infection and the natural recruitment and death rates of vector and host are supposed to be $\mu_{v}$ and $\mu_{h}$, respectively.

The flow of dengue infection from an infected mosquito to a susceptible human starts by the bite of infectious mosquitoes when it sucks the blood of a susceptible host. In both populations, the flow of dengue infection from susceptible state to infected one relies on the biting rate of mosquitoes, the number of susceptible and infectious members of both populations, and the transmission probabilities. In this formulation, $b$ is for the per capita bitting rate of vectors and $\beta_{h_{1}}, \beta_{h_{2}}$ and $\beta_{\nu}$ represent the transmission probabilities from vector to susceptible humans and vice versa. In this formulation, the infection rates per humans and mosquitoes are described by $\left(\frac{b \beta_{h_{1}}}{N_{h}} I_{v}\right),\left(\frac{b \beta_{h_{2}}}{N_{h}} I_{v}\right)$ and $\left(\frac{b \beta_{v}}{N_{h}} I_{h}\right)$, respectively. We assume that a fraction $v$ of the recovered class lose immunity and become susceptible with less transmission probability.

The differential system that represents the flow of dengue infection with the upper mentioned suppositions is specified as

$$
\left\{\begin{array}{l}
\frac{d S_{h_{1}}}{d t}=\mu_{h} N_{h}-\frac{\beta_{h_{1}} b}{N_{h}} S_{h_{1}} I_{v}-\mu_{h} S_{h_{1}}, \\
\frac{d S_{h_{2}}}{d t}=-\frac{\beta_{h_{2}} b}{N_{h}} S_{h_{2}} I_{v}-\mu_{h} S_{h_{2}}+v R_{h}, \\
\frac{d I_{h}}{d t}=\frac{\beta_{h_{1}} b}{N_{h}} S_{h_{1}} I_{v}+\frac{\beta_{h_{2}} b}{N_{h}} S_{h_{2}} I_{v}-\left(\mu_{h}+\gamma_{h}\right) I_{h} \\
\frac{d R_{h}}{d t}=\gamma_{h} I_{h}-\left(v+\mu_{h}\right) R_{h} \\
\frac{d S_{v}}{d t}=\mu_{v} N_{v}-\frac{\beta_{v} b}{N_{h}} S_{v} I_{h}-\mu_{v} S_{v} \\
\frac{d I_{v}}{d t}=\frac{\beta_{v} b}{N_{h}} S_{v} I_{h}-\mu_{v} I_{v}
\end{array}\right.
$$

with appropriate non-negative initial condition, moreover, the total population size of vector and host are

$$
N_{v}=S_{v}+I_{v}, \quad N_{h}=S_{h_{1}}+S_{h_{2}}+I_{h}+R_{h} .
$$

It is well known that there exist periodic outbreaks of dengue infection and fluctuation occurs in the density of the vector population because of environmental factors such as humidity and temperature. Therefore, we introduce periodic transmission rates and seasonality in the vector population to represent more realistically the phenomena of dengue infection. We denote the periodic transmission rates by $\beta_{h_{1}}(t), \beta_{h_{2}}(t)$ and $\beta_{v}(t)$, and we indicate the periodic recruitment rate and natural death rate of mosquitoes by $A(t)$ and $\mu_{\nu}(t)$. Note that here the seasonality in the death rate of mosquitoes is due to the phenology feature in vectors which brings fluctuation in the death rate with time [42]. Further, we introduce an impulsive vaccination mechanism of control and prevention for dengue infection, where vaccination is applied to a fraction $q$ of host population $S_{h_{1}}$, these susceptible hosts shift to the recovery class $R_{h}$ after successful vaccination. Then the system of equations (1) with the above assumptions takes the 
form

$$
\left\{\begin{array}{l}
\frac{d S_{h_{1}}}{d t}=\mu_{h} N_{h}-\frac{b \beta_{h_{1}}(t)}{N_{h}} S_{h_{1}} I_{v}-\mu_{h} S_{h_{1}}, \\
\frac{d S_{h_{2}}}{d t}=-\frac{b \beta_{h_{2}}(t)}{N_{h}} S_{h_{2}} I_{v}-\mu_{h} S_{h_{2}}+v R_{h}, \\
\frac{d I_{h}}{d t}=\frac{b \beta_{h_{1}}(t)}{N_{h}} S_{h_{1}} I_{v}+\frac{b \beta_{h_{2}}(t)}{N_{h}} S_{h_{2}} I_{v}-\left(\mu_{h}+\gamma_{h}\right) I_{h}, \\
\frac{d R_{h}}{d t}=\gamma_{h} I_{h}-\left(v+\mu_{h}\right) R_{h}, \\
\frac{d S_{v}}{d t}=A(t)-\frac{b \beta_{v}(t)}{N_{h}} S_{v} I_{h}-\mu_{v}(t) S_{v}, \\
\frac{d I_{v}}{d t}=\frac{b \beta_{v}(t)}{N_{h}} S_{v} I_{h}-\mu_{v}(t) I_{v}, \\
S_{h_{1}}\left(t^{+}\right)=(1-q) S_{h_{1}}(t), \\
S_{h_{2}}\left(t^{+}\right)=S_{h_{2}}(t), \\
I_{h}\left(t^{+}\right)=I_{h}(t), \\
R_{h}\left(t^{+}\right)=R_{h}(t)+q S_{h_{1}}(t), \\
S_{v}\left(t^{+}\right)=S_{v}(t), \\
I_{v}\left(t^{+}\right)=I_{v}(t),
\end{array}\right\} \quad t=\mathrm{nT}, \mathrm{nT}, \mathrm{n} \in \mathrm{N},
$$

with non-negative initial condition $S_{h_{1}}(0)=S_{h_{1} 0}, S_{h_{2}}(0)=S_{h_{2} 0}, I_{h}(0)=I_{h 0}, R_{h}(0)=R_{h 0}$, $S_{\nu}(0)=S_{\nu 0}$ and $I_{\nu}(0)=I_{\nu 0}$. The above impulsive dynamical model (3) implies

$$
\frac{d N_{v}(t)}{d t}=A(t)-\mu_{v}(t) N_{v}(t)
$$

with non-negative initial condition $N_{\nu}(0)=N_{\nu 0}$. System (4) has a unique positive Tperiodic globally asymptotically stable solution given by

$$
\tilde{N}_{\nu}(t)=\tilde{N}_{\nu}(0) e^{-\int_{0}^{t} \mu_{\nu}(\rho) d \rho}+e^{-\int_{0}^{t} \mu_{\nu}(\rho) d \rho} \int_{0}^{t} A(\rho) e^{\int_{0}^{\rho} \mu_{\nu}(\tau) d \tau} d \rho,
$$

with

$$
\widetilde{N}_{\nu}(0)=\frac{\int_{0}^{\mathrm{T}} A(\rho) e^{\int_{0}^{\rho} \mu_{\nu}(\tau) d \tau} d \rho}{e^{\int_{0}^{\mathrm{T}} \mu_{\nu}(\rho) d \rho}-1} .
$$

In consequence of this, $N_{v}(t) \rightarrow \widetilde{N}_{v}(t)$ as $t$ tends to infinity. Finally, the system of equations (3) with the assumptions of $\beta_{1}(t)=\frac{b \beta_{h_{1}}(t)}{N_{h}}, \beta_{2}(t)=\frac{b \beta_{h_{2}}(t)}{N_{h}}$ and $\beta_{3}(t)=\frac{b \beta_{v}(t)}{N_{h}}$, takes the form

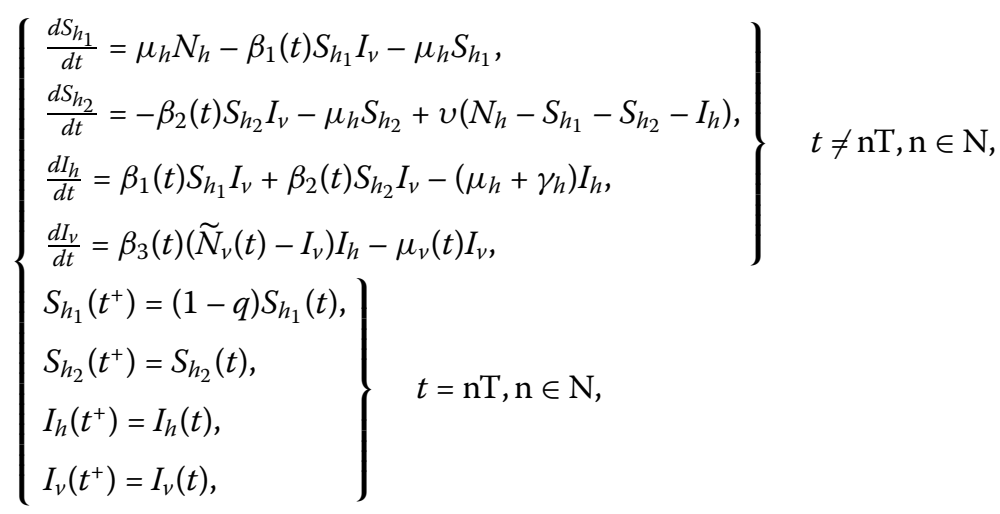


with non-negative initial condition $S_{h_{1}}(0)=S_{h_{1} 0}, S_{h_{2}}(0)=S_{h_{2} 0}, I_{h}(0)=I_{h 0}$ and $I_{v}(0)=$ $I_{\nu 0}$.

To analyze the impulsive system (5), we take the general impulsive differential system

$$
\begin{aligned}
& \frac{d \mathrm{y}(\mathrm{t})}{d \mathrm{t}}=b-d \mathrm{y}(\mathrm{t}), \quad \mathrm{t} \neq \mathrm{nT}, \mathrm{n} \in \mathrm{N}, \\
& \mathrm{y}\left(\mathrm{t}^{+}\right)=(1-\phi) \mathrm{y}(\mathrm{t}), \quad \mathrm{t}=\mathrm{nT}, \mathrm{n} \in \mathrm{N},
\end{aligned}
$$

where $b, d$ are positive and $0<\phi<1$. Here, we present that the impulsive dynamical system (6) admits a positive periodic, and globally asymptotically stable solution.

Lemma 2.1 The impulsive dynamical system (6) has a positive periodic globally asymptotically stable solution

$$
\widetilde{\mathrm{y}}(\mathrm{t})=\frac{b}{d}+\left(\mathrm{y}^{*}-\frac{b}{d}\right) e^{-d(\mathrm{t}-\mathrm{nT})}, \quad \mathrm{t} \in(\mathrm{nT},(\mathrm{n}+1) \mathrm{T}]
$$

where

$$
\mathrm{y}^{*}=\frac{b(1-\phi)\left(1-e^{-d \mathrm{~T}}\right)}{d\left[1-(1-\phi) e^{-d \mathrm{~T}}\right]} .
$$

Proof To obtain the required result, we integrate the first equation of the dynamical system (6) at pulses, and we get

$$
\widetilde{\mathrm{y}}(\mathrm{t})=\frac{b}{d}+\left(\mathrm{y}(\mathrm{nT})-\frac{b}{d}\right) e^{-d(\mathrm{t}-\mathrm{nT})}, \quad \mathrm{t} \in(\mathrm{nT},(\mathrm{n}+1) \mathrm{T}],
$$

where $\mathrm{y}(\mathrm{nT})$ denotes the base value at $\mathrm{nT}$. In the next step, we solve the last equation of the impulsive dynamical system (6) and obtain

$$
\mathrm{y}((\mathrm{n}+1) \mathrm{T})=(1-\phi)\left[\frac{b}{d}+\left(\mathrm{y}(\mathrm{nT})-\frac{b}{d}\right) e^{-d \mathrm{~T}}\right]=g(\mathrm{y}(\mathrm{nT})),
$$

where $g(\mathrm{y})=(1-\phi)\left[\frac{b}{d}+\left(\mathrm{y}-\frac{b}{d}\right) e^{-d \mathrm{~T}}\right]$. Furthermore, it is effortless to show that (8) has a unique positive solution given by $\mathrm{y}^{*}=\left[\frac{b(1-\phi)\left(1-e^{-d \mathrm{~T}}\right)}{d\left(1-(1-\phi) e^{-d \mathrm{~T}}\right)}\right]$, which satisfies the condition $\mathrm{y}<$ $g(\mathrm{y})<\mathrm{y}^{*}$, if $0<\mathrm{y}<\mathrm{y}^{*}$ and satisfies the condition $\mathrm{y}^{*}<g(\mathrm{y})<\mathrm{y}$ if $\mathrm{y}>\mathrm{y}^{*}$. Evidently, $y^{*}$ is globally asymptotically stable for Eq. (8) by [43]. Consequently, we have a positive periodic globally asymptotically stable solution of the impulsive system (6), given by

$$
\widetilde{\mathrm{y}}(\mathrm{t})=\frac{b}{d}+\left(\mathrm{y}^{*}-\frac{b}{d}\right) e^{-d(\mathrm{t}-\mathrm{nT})}, \quad \mathrm{t} \in(\mathrm{nT},(\mathrm{n}+1) \mathrm{T}]
$$

with

$$
\mathrm{y}^{*}=\frac{b(1-\phi)\left(1-e^{-d \mathrm{~T}}\right)}{d\left[1-(1-\phi) e^{-d \mathrm{~T}}\right]} .
$$

This completes the proof. 
Next, we present the following concept for the solution of a linear system of the form $\mathrm{y}^{\prime}=G(t) \mathrm{y}$. Let $\mathrm{w}_{1}, \mathrm{w}_{2} \in \mathbb{R}^{n}$, where $\left(\mathbb{R}^{n}, \mathbb{R}_{+}^{n}\right)$ is a standard order $\mathrm{n}$-dimensional Euclidean space and $\|\cdot\|$ is the norm defined on $\left(\mathbb{R}^{n}, \mathbb{R}_{+}^{n}\right)$. Further, we take $\mathrm{w}_{1} \geq \mathrm{w}_{2}$, if $\mathrm{w}_{1}-\mathrm{w}_{2} \in \mathbb{R}_{+}^{n}$; $\mathrm{w}_{1}>\mathrm{w}_{2}$, if $\mathrm{w}_{1}-\mathrm{w}_{2} \in \mathbb{R}_{+}^{n} \backslash 0$; and $\mathrm{w}_{1} \gg \mathrm{w}_{2}$, if $\mathrm{w}_{1}-\mathrm{w}_{2} \in \operatorname{Int}\left(\mathbb{R}_{+}^{n}\right)$. Here, we assume a matrix function $G(t)$ of order $n$, which is irreducible, cooperative, continuous and periodic with a positive period $\omega$. Take the linear system $\mathrm{y}^{\prime}=G(t)$ y having a fundamental matrix solution $\Phi_{G(\cdot)}(t)$ and $r\left(\Phi_{G(\cdot)}(\omega)\right)$ represents the highest eigenvalue of $\Phi_{G(\cdot)}(\omega)$ in magnitude. Then the Perron-Frobenius theorem implies that $r\left(\Phi_{G(\cdot)}(\omega)\right)$ is the principal eigenvalue of the solution matrix $\Phi_{G(\cdot)}(\omega)$. The following lemma is introduced for the further analysis of our impulsive system.

Lemma $2.2([44,45])$ Let $\psi=\frac{1}{\omega} \ln r\left(\Phi_{G(\cdot)}(\omega)\right)$. Then the linear system $\mathrm{y}^{\prime}=G(t) \mathrm{y}$ has a solution $e^{\psi t} \mathrm{u}(\mathrm{t})$, where $\mathrm{u}(\mathrm{t})$ is a $\omega$-periodic and positive function.

\section{Threshold dynamics}

The existence of the disease-free periodic solution of impulsive dynamical model (5) and its stability will be studied in this section of the article. Suppose both populations are free of infections, more specifically there is no infection in host and vector populations, that is, $I_{h}(t)=I_{v}(t)=0$, then the impulsive system (5) becomes

$$
\left\{\begin{array}{l}
\left.\begin{array}{l}
\frac{d S_{h_{1}}}{d t}=\mu_{h} N_{h}-\mu_{h} S_{h_{1}}, \\
\frac{d S_{h_{2}}}{d t}=-\mu_{h} S_{h_{2}}+v\left(N_{h}-S_{h_{1}}-S_{h_{2}}\right),
\end{array}\right\} \quad t \neq \mathrm{nT}, \mathrm{n} \in \mathrm{N}, \\
S_{h_{1}}\left(t^{+}\right)=(1-q) S_{h 1}(t), \\
S_{h_{2}}\left(t^{+}\right)=S_{h_{2}}(t),
\end{array}\right\} \quad t=\mathrm{nT}, \mathrm{n} \in \mathrm{N},
$$

with proper non-negative initial conditions. The following result is concerned with the positive periodic solution of the disease-free system (9) and its global asymptotical stability.

Theorem 3.1 The impulsive dynamical system (9) admits a positive periodic, and globally asymptotically stable solution.

Proof To obtain the result, firstly we take the portion of $S_{h_{1}}$ of the impulsive system (9), that is

$$
\begin{cases}\frac{d S_{h_{1}}}{d t}=\mu_{h} N_{h}-\mu_{h} S_{h_{1}}, & t \neq \mathrm{nT}, \mathrm{n} \in \mathrm{N}, \\ S_{h_{1}}\left(t^{+}\right)=(1-q) S_{h_{1}}(t), & t=\mathrm{nT}, \mathrm{n} \in \mathrm{N} .\end{cases}
$$

Obviously, the impulsive system (10) admits a positive periodic globally asymptotically stable solution by Lemma 2.1, given by

$$
\widetilde{S}_{h_{1}}(t)=N_{h}+\left(S_{h_{1}}^{*}-N_{h}\right) e^{-\mu_{h}(t-\mathrm{nT})}, \quad t \in(\mathrm{nT},(\mathrm{n}+1) \mathrm{T}],
$$

with

$$
S_{h_{1}}^{*}=\frac{N_{h}(1-q)\left(1-e^{-\mu_{h} \mathrm{~T}}\right)}{\left[1-(1-q) e^{-\mu_{h} \mathrm{~T}}\right]} .
$$


Secondly, we take the portion of $S_{h_{2}}$ of the impulsive system (9), that is

$$
\begin{cases}\frac{d S_{h_{2}}(t)}{d t}=v\left(N_{h}-\widetilde{S}_{h_{1}}(t)\right)-\left(v+\mu_{h}\right) S_{h_{2}}(t), & t \neq \mathrm{nT}, \mathrm{n} \in \mathrm{N} \\ S_{h_{2}}\left(t^{+}\right)=S_{h_{2}}(t), & t=\mathrm{nT}, \mathrm{n} \in \mathrm{N}\end{cases}
$$

The above-mentioned system (12) also admits a positive periodic globally asymptotically stable solution, given by

$$
\begin{aligned}
& \widetilde{S}_{h_{2}}(t)=e^{-\left(v+\mu_{h}\right)(t-\mathrm{nT})}\left[S_{h_{2}}^{*}+\int_{\mathrm{nT}}^{t} v\left(N_{h}-\widetilde{S}_{h_{1}}(\rho)\right) e^{\left(v+\mu_{h}\right)(t-\mathrm{nT})} d \rho\right], \\
& t \in(\mathrm{nT},(\mathrm{n}+1) \mathrm{T}]
\end{aligned}
$$

with

$$
S_{h_{2}}^{*}=\frac{\int_{\mathrm{nT}}^{(\mathrm{n}+1) \mathrm{T}} v\left(N_{h}-\widetilde{S}_{h_{1}}(\rho)\right) e^{\left(v+\mu_{h}\right) \rho} d \rho}{e^{\left(v+\mu_{h}\right) \mathrm{T}}-1} .
$$

As a result, the impulsive system (9) has a positive periodic globally asymptotically stable solution, i.e., $\left(\widetilde{S}_{h_{1}}(t), \widetilde{S}_{h_{2}}(t)\right)$. This completes the proof.

Theorem 3.1 ensures that the impulsive dynamical system (5) has a unique positive periodic disease-free solution $\left(\tilde{S}_{h_{1}}(t), \tilde{S}_{h_{2}}(t), 0,0\right)$ on every impulsive interval. Next, to investigate the stability results of disease-free periodic solution of impulsive system (5), let us take the linear system $\mathrm{y}^{\prime}=G(t) \mathrm{y}$, where $G(t)$ is a matrix of order $n$ and assume that $\Phi_{G(\cdot)}(t)$ is its fundamental solution. Also, the spectral radius of $\Phi_{G(\cdot)}(\omega)$ is assumed to be $r\left(\Phi_{G(\cdot)}(\omega)\right)$, then the local stability result of the disease-free solution can be obtained by taking smallamplitude perturbations of the solutions. After small perturbations, the linearizing system of (5) at the infection-free periodic solution is

$$
\begin{aligned}
& \mathrm{y}^{\prime}(t)=G(t) \mathrm{y}(t), \quad t \neq \mathrm{nT}, \mathrm{n} \in \mathrm{N} \\
& \mathrm{y}\left(t^{+}\right)=H \mathrm{y}(t), \quad t=\mathrm{nT}, \mathrm{n} \in \mathrm{N}
\end{aligned}
$$

in this linear system, we have

$$
G(t)=\left[\begin{array}{cc}
G_{1} & G_{2}(t) \\
O & \mathrm{~F}(t)-\mathrm{V}(t)
\end{array}\right], \quad H(t)=\left[\begin{array}{cc}
H_{1} & 0 \\
0 & I
\end{array}\right],
$$

with

$$
\begin{aligned}
& G_{1}=\left[\begin{array}{cc}
-\mu_{h} & 0 \\
-v & -\left(\mu_{h}+v\right)
\end{array}\right], \quad G_{2}(t)=\left[\begin{array}{cc}
0 & -\beta_{1}(t) \widetilde{S}_{h_{1}}(t) \\
-v & -\beta_{2}(t) \widetilde{S}_{h_{2}}(t)
\end{array}\right], \\
& O=\left[\begin{array}{ll}
0 & 0 \\
0 & 0
\end{array}\right], \quad H_{1}=\left[\begin{array}{cc}
1-q & 0 \\
0 & 1
\end{array}\right], \quad \mathrm{V}(t)=\left[\begin{array}{cc}
\left(\mu_{h}+\gamma_{h}\right) & 0 \\
0 & \mu_{v}(t)
\end{array}\right], \quad \text { and } \\
& \mathrm{F}(t)=\left[\begin{array}{cc}
0 & \beta_{1}(t) \widetilde{S}_{h_{1}}(t)+\beta_{2}(t) \widetilde{S}_{h_{2}}(t) \\
\beta_{3}(t) \tilde{N}_{v}(t) & 0
\end{array}\right] .
\end{aligned}
$$


Let $\Phi_{G}(t)=\left(\Phi_{i j}\right)_{1 \leq i, j \leq 2}$ be the fundamental solution of linear system y $\mathrm{y}^{\prime}(t)=G(t) \mathrm{y}(t)$. Consequently, we have $\Phi_{G}^{\prime}(t)=G(t) \Phi_{G}(t)$ with appropriate initial value $\Phi_{G}(0)=E_{4}$. Further computation implies that

$$
\Phi_{G}(t)=\left[\begin{array}{cc}
e^{G_{1} t} & \Phi_{12}(t) \\
0 & \Phi_{\mathrm{F}(t)-\mathrm{V}(t)}(t)
\end{array}\right],
$$

then we obtain

$$
H \Phi_{G}(\mathrm{~T})=\left[\begin{array}{cc}
H_{1} e^{G_{1} T} & H_{1} \Phi_{12}(\mathrm{~T}) \\
0 & \Phi_{(\mathrm{F}(t)-\mathrm{V}(t))}(\mathrm{T})
\end{array}\right] .
$$

It can be noticed that $r\left(H_{1} e^{G_{1} T}\right)<1$. Denote the basic reproduction number

$$
\mathrm{R}_{0} \triangleq r\left(\Phi_{(\mathrm{F}-\mathrm{V})(\cdot)(t)}(\mathrm{T})\right)
$$

therefore, based on Floquet theory, we obtain the following conclusion.

Theorem 3.2 The infection-free periodic solution of the impulsive dynamical system (5) is locally asymptotically stable if $\mathrm{R}_{0}$ less than 1 , and is unstable in other cases.

Note that here $R_{0}$ works as a threshold (endemic indicator) for the impulsive system (5) such that, if $R_{0}$ is less than 1 , then an infected individual generate less than an infected individual and the infection vanishes. In the case that $R_{0}$ greater than 1 , this implies that an infected individual generates more infected individuals in the community [46].

Theorem 3.3 The infection-free periodic solution of impulsive dynamical system (5) is globally asymptotically stable for $\mathrm{R}_{0}<1$.

Proof To obtain the result, we take the solution $\varphi(t, x)=\left(S_{h_{1}}(t), S_{h_{2}}(t), I_{h}(t), I_{v}(t)\right)$ of the impulsive system (5), where $x \in R_{+}^{4}$. By the non-negativity of $I_{h}(t)$ and $I_{v}(t)$, we have

$$
\begin{cases}\frac{d S_{h_{1}}}{d t} \leq \mu_{h} N_{h}-\mu_{h} S_{h_{1}}, & t \neq \mathrm{nT}, \mathrm{n} \in \mathrm{N}, \\ S_{h_{1}}\left(t^{+}\right)=(1-q) S_{h_{1}}(t), & t=\mathrm{nT}, \mathrm{n} \in \mathrm{N},\end{cases}
$$

taking the auxiliary system

$$
\begin{cases}\frac{d x_{1}}{d t}=\mu_{h} N_{h}-\mu_{h} x_{1}, & t \neq \mathrm{nT}, \mathrm{n} \in \mathrm{N} \\ x_{1}\left(t^{+}\right)=(1-q) x_{1}(t), & t=\mathrm{nT}, \mathrm{n} \in \mathrm{N} .\end{cases}
$$

Obviously, the impulsive system (16) admits a positive periodic globally asymptotically stable solution by Lemma 2.1 , say $\widetilde{x}_{1}(t)$, that is, $x_{1}(t)$ converges to $\widetilde{x}_{1}(t)$, as $t$ tends to infinity. Further, the comparison theorem of IDEs implies that $S_{h_{1}}(t) \leq x_{1}(t)$, similarly we can justify that $S_{h_{2}}(t) \leq x_{2}(t)$. Next, we define the solution $S(t)=\left(S_{h_{1}}(t), S_{h_{2}}(t)\right)^{T}$ and $x(t)=\left(x_{1}(t), x_{2}(t)\right)^{T}$ of the infection-free impulsive system (9), with $S(0)=x(0)$. It is clear that the $\tau$-periodic solution is globally asymptotically stable for the infection-free system (9) by Theorem 3.1 , say $\widetilde{S}(t)=\left(\widetilde{S}_{h_{1}}(t), \widetilde{S}_{h_{2}}(t)\right)^{T}$. To be more specific, the solution 
$x(t) \rightarrow \widetilde{S}(t)$ as $\mathrm{t}$ tends to infinity. We fix $\xi>0$. Then we can find $t_{1}>0$ in such a manner that $x_{1}(t) \leq \widetilde{S}_{h_{1}}(t)+\xi$ and $x_{2}(t) \leq \widetilde{S}_{h_{2}}(t)+\xi$ for all $t \geq t_{1}$. Thus, for $t \geq t_{1}$, we have $S_{h_{1}}(t) \leq \widetilde{S}_{h_{1}}(t)+\xi$ and $S_{h_{2}}(t) \leq \widetilde{S}_{h_{2}}(t)+\xi$. By system (5), we have

$$
\left\{\begin{array}{l}
\frac{d I_{h}}{d t} \leq\left(\beta_{1}(t)\left(\widetilde{S}_{h_{1}}(t)+\xi\right)+\beta_{2}(t)\left(\widetilde{S}_{h_{2}}(t)+\xi\right)\right) I_{v}-\left(\mu_{h}+\gamma_{h}\right) I_{h}, \\
\frac{d I_{v}}{d t} \leq \beta_{3}(t) \widetilde{N}_{v}(t) I_{h}-\mu_{v}(t) I_{v} .
\end{array}\right.
$$

Taking the auxiliary system

$$
\left\{\begin{array}{l}
\frac{d w_{1}}{d t}=\left(\beta_{1}(t)\left(\widetilde{S}_{h_{1}}(t)+\xi\right)+\beta_{2}(t)\left(\widetilde{S}_{h_{2}}(t)+\xi\right)\right) w_{2}-\left(\mu_{h}+\gamma_{h}\right) w_{1}, \\
\frac{d w_{2}}{d t}=\beta_{3}(t) \widetilde{N}_{v}(t) w_{1}-\mu_{v}(t) w_{2},
\end{array}\right.
$$

system (18) is converted into

$$
\mathrm{w}^{\prime}=(\mathrm{F}(t)-\mathrm{V}(t)) \mathrm{w}+\xi W(t) \mathrm{w}
$$

where $\mathrm{w}$ is a vector of the form $\mathrm{w}=\left(\mathrm{w}_{1}, \mathrm{w}_{2}\right)^{\mathrm{T}}$ and

$$
W(t)=\left[\begin{array}{cc}
0 & \beta_{1}(t)+\beta_{2}(t) \\
0 & 0
\end{array}\right]
$$

Based on Lemma 2.2, the linear differential system (19) has a solution, that is, $e^{\mu_{1} t} u(t)$, where $\mu_{1}=\frac{1}{\mathrm{~T}} \ln r\left(\Phi_{(\mathrm{F}-\mathrm{V})(\cdot)(t)+\xi W(t)}(\mathrm{T})\right)$ and the vector $u(t)=\left(u_{1}(t), u_{2}(t)\right)^{T}$ is a positive Tperiodic function. Choose $t_{2}>t_{1}$ and a small number $\alpha>0$ in such a way that $\mathrm{w}\left(t_{2}\right) \leq$ $\alpha u(0)$. Consequently we obtain

$$
\mathrm{w}(t) \leq \alpha e^{\mu_{1}\left(t-t_{2}\right)} u\left(t-t_{2}\right), \quad t \geq t_{2} .
$$

Applying Theorem B.1 presented in [47], we have

$$
\left(I_{h}(t), I_{v}(t)\right)^{T} \leq \mathrm{w}(t) \leq \alpha e^{\mu_{1}\left(t-t_{2}\right)} u\left(t-t_{2}\right), \quad t \geq t_{2} .
$$

While $\mathrm{R}_{0}<1$, one can select a small $\xi$ in such a way that $r\left(\Phi_{(\mathrm{F}(t)-\mathrm{V}(t))(\cdot)+\xi W(t)}(\mathrm{T})\right)<1$. Hence, we obtain $\mu_{1}<0$. As a result $\mathrm{w}(t) \rightarrow 0$ as $t$ approaches infinity, it shows that $\left(I_{h}(t), I_{v}(t)\right)^{T} \rightarrow 0$ as $t$ approaches infinity. As a result, we obtain $\lim _{t \rightarrow \infty} S_{h_{1}}(t)=\widetilde{S}_{h_{1}}(t)$ and $\lim _{t \rightarrow \infty} S_{h_{2}}(t)=\widetilde{S}_{h_{2}}(t)$ from the impulsive system (5). Thus, the solution of the impulsive system is globally attractive. As a consequence, the disease-free periodic solution of impulsive system (5) is GAS for $R_{0}<1$. This completes the proof.

Remark The stability result presented in Theorem 3.3 exhibits the threshold dynamics and agrees with the epidemiological mechanism, where the infection will vanish if the average number infected by an infective individual in his infectious period is less than 1, but the infection will remain in the community if the average number is greater than 1 . Moreover, the stability of the infection-free solution means that the host population converges to the periodic functions given by (11) and (13), while the vector population tends to zero. 
Theorem 3.4 The infection in the impulsive system (5) is uniformly persistent if $\mathrm{R}_{0}>1$, in other words, there is $\kappa>0$ such that $\lim _{t \rightarrow \infty} \inf I_{h}(t)>\kappa, \lim _{t \rightarrow \infty} \inf I_{v}(t)>\kappa$.

Proof To obtain the required result, we will first provide evidence that we can find a constant $\kappa>0$, in such a way as to make

$$
\lim _{t \rightarrow \infty} \sup I_{h}(t)>\kappa, \quad \lim _{t \rightarrow \infty} \sup I_{\nu}(t)>\kappa .
$$

In conflict with this, we take the assumption as regards time $t$ in such a manner that $I_{h}(t)<$ $\kappa$ and $I_{v}(t)<\kappa$, for all $t \geq t_{1}$. From system (5), we have

$$
\begin{cases}\frac{d S_{h_{1}}}{d t} \geq \mu_{h} N_{h}-\beta_{1}(t) S_{h_{1}} \kappa-\mu_{h} S_{h_{1}}, & t \neq \mathrm{nT}, \mathrm{n} \in \mathbb{N}, \\ S_{h_{1}}\left(t^{+}\right)=(1-q) S_{h_{1}}(t), & t=\mathrm{nT}, \mathrm{n} \in \mathbb{N} .\end{cases}
$$

We take the auxiliary system

$$
\begin{aligned}
& \frac{d x_{1}}{d t}=\mu_{h} N_{h}-\left(\beta_{1}(t) \kappa+\mu_{h}\right) x_{1}, \quad t \neq \mathrm{nT}, \mathrm{n} \in \mathbb{N}, \\
& x_{1}\left(t^{+}\right)=(1-q) x_{1}(t), \quad t=\mathrm{nT}, \mathrm{n} \in \mathbb{N}
\end{aligned}
$$

The above-mentioned impulsive system (21) has a positive $\omega$-periodic globally asymptotically stable solution,

$$
\widetilde{x}_{1}(t)=e^{-\int_{\mathrm{nT}}^{t}\left(\beta_{1}(\rho) \kappa+\mu_{h}\right) d \rho}\left(x_{1}^{*}+\int_{\mathrm{nT}}^{t} \mu_{h} N_{h} e^{\int_{\mathrm{nT}}^{\rho}\left(\beta_{1}(\tau) \kappa+\mu_{h}\right) d \tau} d \rho\right), \quad t \in(\mathrm{nT},(\mathrm{n}+1) \mathrm{T}],
$$

where

$$
x_{1}^{*}=(1-q)\left[\frac{\int_{\mathrm{nT}}^{(\mathrm{n}+1) \mathrm{T}} \mu_{h} N_{h} e^{\int_{\mathrm{nT}}^{\rho}\left(\beta_{1}(\tau) k+\mu_{h}\right) d \tau} d \rho}{e^{\int_{\mathrm{nT}}^{(\mathrm{n}+1) \mathrm{T}}\left(\beta_{1}(\rho) \kappa+\mu_{h}\right) d \rho}-(1-q)}\right] .
$$

In other words, $x_{1}(t) \rightarrow \widetilde{x}_{1}(t)$, as $t$ tends to infinity. The comparison theorem of IDEs implies that $S_{h_{1}}(t) \geq x_{1}(t)$, similarly we can justify that $S_{h_{2}}(t) \geq x_{2}(t)$. At the moment $\lim _{\kappa \rightarrow 0}\left(\widetilde{x}_{1}, \widetilde{x}_{2}\right)=\left(\widetilde{S}_{h_{1}}, \widetilde{S}_{h_{2}}\right)$. As a consequence of this, there exists small $\kappa_{1}$ for any $\xi_{1}>0$ such that $\widetilde{x}_{1} \geq \widetilde{S}_{h_{1}}-\xi_{1}$ and $\widetilde{x}_{2} \geq \widetilde{S}_{h_{2}}-\xi_{1}$ for $\kappa<\kappa_{1}$. Using a comparison analysis, there exist $t_{2} \geq t_{1}$ and $\xi_{2}>0$, in such a manner that

$$
S_{h_{1}}(t) \geq x_{1}(t) \geq \widetilde{x}_{1}-\xi_{2} \geq \widetilde{S}_{h_{1}}-\xi_{1}-\xi_{2}
$$

and

$$
S_{h_{2}}(t) \geq x_{2}(t) \geq \widetilde{x}_{2}-\xi_{2} \geq \widetilde{S}_{h_{2}}-\xi_{1}-\xi_{2} .
$$

Again from the dynamical system (5), we have

$$
\begin{aligned}
& \frac{d I_{h}}{d t} \geq \beta_{1}(t)\left(\tilde{S}_{h_{1}}(t)-\xi_{1}-\xi_{2}\right) I_{v}+\beta_{2}(t)\left(\tilde{S}_{h_{2}}(t)-\xi_{1}-\xi_{2}\right) I_{\nu}-\left(\mu_{h}+\gamma_{h}\right) I_{h}, \\
& \frac{d I_{v}}{d t} \geq \beta_{3}(t)\left(\tilde{N}_{v}(t)-\kappa\right) I_{h}-\mu_{v}(t) I_{v} .
\end{aligned}
$$


We take the auxiliary system

$$
w^{\prime}=(F(t)-V(t)) w-M(t) w
$$

where $w=\left(w_{1}, w_{2}\right)^{\mathrm{T}}$. In the premises of Lemma 2.2, we claim that we can find a T-periodic and positive function $v(t)=\left(v_{1}(t), v_{2}(t)\right)$ so that $e^{\mu_{2} t} v(t)$ is a solution of system (23) with $\mu_{2}=\frac{1}{\mathrm{~T}} \ln r\left(\Phi_{(\mathrm{F}-\mathrm{V})(\cdot)(t)-M(t)}(\mathrm{T})\right)$. Select $t_{3}>t_{2}$ and an $\eta>0$ small enough in such a manner that $w\left(t_{2}\right) \geq \eta v(0)$. We get

$$
w(t) \geq \eta e^{\mu_{2}\left(t-t_{2}\right)} v\left(t-t_{2}\right), \quad t \geq t_{3} .
$$

Applying the standard comparison theorem presented in [47], we have

$$
\left(I_{h}(t), I_{v}(t)\right)^{T} \geq w(t) \geq \eta e^{\mu_{2}\left(t-t_{2}\right)} v\left(t-t_{2}\right), \quad t \geq t_{3} .
$$

As $r\left(\Phi_{(\mathrm{F}-\mathrm{V})}(\cdot)(t)(\mathrm{T})\right)>1$ and $r\left(\Phi_{(\mathrm{F}-\mathrm{V})}(\cdot)(t)-M(t)(\mathrm{T})\right)$ is continuous for small $\xi_{1}$ and $\xi_{2}$, we may choose $\xi_{1}, \xi_{2}>0$ in such a way that $r\left(\Phi_{(\mathrm{F}-\mathrm{V})(\cdot)-M}(\mathrm{~T})\right)>1$. As a result we obtain $\mu_{2}>0$. Because of this $w(t)$ converges to infinity as $t \rightarrow \infty$, consequently $\left(I_{h}(t), I_{v}(t)\right)^{T} \rightarrow \infty$ as $t \rightarrow \infty$, which conflicts the boundedness of $I_{h}$ and $I_{v}$. Thus we obtain the proof of the claim, that is, $\lim _{t \rightarrow \infty} \sup I_{h}(t) \geq \kappa$ and $\lim _{t \rightarrow \infty} \sup I_{v}(t) \geq \kappa$. Furthermore, we have the following two possibilities from the claim:

(a) $I_{h}(t)>\kappa$ and $I_{\nu}(t)>\kappa$ for large $t$;

(b) oscillations of $I_{h}(t)$ and $I_{\nu}(t)$ about $\kappa$ for large $t$.

If case (a) is satisfied, then the required proof is obtained. Next, we focus on case (b). As $\lim _{t \rightarrow \infty} \sup I_{h}(t) \geq \kappa$ and $\lim _{t \rightarrow \infty} \sup I_{\nu}(t) \geq \kappa$, we can find a $t_{1} \in\left(\mathrm{n}_{1} \mathrm{~T},\left(\mathrm{n}_{1}+1\right) \mathrm{T}\right]$ in such a way that $I_{h}\left(t_{1}\right) \geq \kappa$ and $I_{v}\left(t_{1}\right) \geq \kappa$. The above discussion implies that there exists $t_{2} \in\left(\mathrm{n}_{2} \mathrm{~T},\left(\mathrm{n}_{2}+1\right) \mathrm{T}\right]$, such that $I_{h}\left(t_{2}\right) \geq \kappa$ and $I_{v}\left(t_{2}\right) \geq \kappa$, where $\mathrm{n}_{2}-\mathrm{n}_{1} \geq 0$ is finite. We will now observe the solution of the impulsive system (5) in the interval $\left[t_{1}, t_{2}\right]$ :

$$
I_{h}^{\prime}=\beta_{1}(t) S_{h_{1}} I_{v}+\beta_{2}(t) S_{h_{2}} I_{v}-\left(\mu_{h}+\gamma_{h}\right) I_{h} \geq-\left(\mu_{h}+\gamma_{h}\right) I_{h} .
$$

Consequently, we get

$$
I_{h}(t) \geq I_{h}\left(t_{1}\right) e^{-\left(\mu_{h}+\gamma_{h}\right)\left(t-t_{1}\right)} \geq \kappa e^{-\left(\mu_{h}+\gamma_{h}\right)\left(t_{2}-t_{1}\right)} \geq \kappa e^{-\left(\mu_{h}+\gamma_{h}\right)\left(\mathrm{n}_{2}-\mathrm{n}_{1}\right) \mathrm{T}} .
$$

Moreover,

$$
I_{v}^{\prime}=\beta_{3}(t)\left(\widetilde{N}_{v}(t)-I_{v}\right) I_{h}-\mu_{v}(t) I_{v} \geq-\mu_{v}(t) I_{v}
$$

which gives

$$
I_{\nu}(t) \geq I_{\nu}\left(t_{1}\right) e^{-\int_{t_{1}}^{t} \mu_{\nu}(s) d s} \geq \kappa e^{-\int_{t_{1}}^{t_{2}} \mu_{\nu}(s) d s} \geq \kappa e^{-\int_{\mathrm{n}_{1} \mathrm{~T}}^{\mathrm{n}_{2} \mathrm{~T}} \mu_{\nu}(t) d t} .
$$

Let $\kappa_{1}=\min \left\{\kappa e^{-\int_{\mathrm{n}_{1} \mathrm{~T}}^{\mathrm{n}_{2} \mathrm{~T}} \mu_{\nu}(t) d t}, \kappa e^{-\left(\mu_{h}+\gamma_{h}\right)\left(\mathrm{n}_{2}-\mathrm{n}_{1}\right) \mathrm{T}}\right\}$, then $\kappa_{1}>0$ cannot be infinitely small and $\mathrm{n}_{2}-\mathrm{n}_{1} \geq 0$ is finite. We have $I_{h}(t) \geq \kappa_{1}>0$ and $I_{v}(t) \geq \kappa_{1}>0$. 
Taking the same steps for $t>t_{2}$, we obtain another non-infinitesimal positive $\kappa_{2}$. As a consequence of this, we get the sequence $\left\{\kappa_{j}\right\}$, where $\kappa_{k}=\min \left\{\kappa e^{-\int_{n_{\mathrm{K}} \mathrm{T}}^{\mathrm{n}_{\mathrm{k}+1} \mathrm{~T}} \mu_{\nu}(t) d t}\right.$, $\left.\kappa e^{-\left(\mu_{h}+\gamma_{h}\right)\left(\mathrm{n}_{\mathrm{k}+1}-\mathrm{n}_{\mathrm{k}}\right) \mathrm{T}}\right\}$ for $j=1,2, \ldots, k, \ldots$ is non-infinitesimal, as the term $\mathrm{n}_{\mathrm{k}+1}-\mathrm{n}_{\mathrm{k}} \geq 0$ is finite. In this case, the solution of system (5) $I_{h}(t) \geq \kappa_{k}>0$ and $I_{\nu}(t) \geq \kappa_{k}>0$ holds true in the interval $\left[t_{k}, t_{k+1}\right]$ of time, where $t_{k} \in\left(\mathrm{n}_{\mathrm{k}} \mathrm{T}, \mathrm{n}_{\mathrm{k}+1} \mathrm{~T}\right], t_{k+1} \in\left(\mathrm{n}_{\mathrm{k}+1} \mathrm{~T},\left(\mathrm{n}_{\mathrm{k}+1}+1\right) \mathrm{T}\right]$. Let $\kappa^{*}=\min _{j}\left(\kappa_{j}\right)=\kappa_{l}>0, l \in N, \kappa_{l} \in\left\{\kappa_{j}\right\}$ for $j=1,2, \ldots$, hence $I_{h}(t) \geq \kappa^{*}>0$ and $I_{v}(t) \geq \kappa^{*}>0$ for all $t \geq t_{1}$. This completes the proof.

\section{Numerical results}

In this part of the article, we will analyze and investigate the dengue disease transmission model (5) numerically to conceptualize the transmission pathway of dengue infection in the proposed impulsive system, and to scrutinize the consequences of impulsive vaccination on the transmission dynamics. The periodic transmission probabilities from vector to host and vice versa are taken as $\beta_{h_{1}}(t)=\alpha_{1}\left(1+\delta_{1} \cos (2 \pi t)\right), \beta_{h_{2}}(t)=\alpha_{2}\left(1+\delta_{2} \cos (2 \pi t)\right)$ and $\beta_{v}(t)=\alpha_{3}\left(1+\delta_{3} \cos (2 \pi t)\right)$, respectively.

To demonstrate the influence of pulse vaccination on the transmission of dengue infection numerically, we use the values of parameter for simulation purpose inventoried in Table 1 and select the state variables for simulation purpose as $S_{v}(0)=18,000, I_{v}(0)=3000$, $S_{h 1}(0)=30,000, S_{h 2}(0)=20,000, I_{h}(0)=1000$ and $R_{h}(0)=20,000$. Furthermore, we choose the values $\alpha_{1}=0.750, \alpha_{2}=0.300, \alpha_{3}=0.750, \delta_{1}=0.05, \delta_{2}=0.03$, and $\delta_{3}=0.01$ to conceptualize the dynamical behavior of our impulsive system, moreover, we assumed constant birth rates for simplicity in our simulations. In Fig. 1, we illustrate the moment path of infected host individuals $I_{h}(t)$ and infected vector individuals $I_{v}(t)$ with fixed-moment pulse vaccination and the vaccination strength $q=0.25$, which demonstrates the endemic intensity of dengue infection in both species. To examine the potency of the vaccination rate $q$, we increase the vaccination rate from 0.25 to 0.75 , as shown in Fig. 2 . We observe that the vaccination measure has a great influence on the system, which means that pulse vaccination can efficiently control infected individuals and can effectively prevent a dengue outbreak. To be more specific, it can be noticed that after implementing the vaccination strategy, the lower level of infected classes in vector and host decreases. Numerical results predict that the vaccination rate $q$ has a great influence on the infection and can successfully decrease the outbreak.

Now we determine the influence of different parameters on the threshold parameter $\mathrm{R}_{0}$ of system (5). We firstly examine the influence of the biting rate of mosquitoes on the threshold parameter, illustrated in Fig. 3(a). It shows that changing the biting rate $b$ from

Table 1 Parameters values with interpretation used in numerical simulations

\begin{tabular}{llll}
\hline Parameter & Interpretation & Values & References \\
\hline$\mu_{h}$ & Recruitment and natural mortality rate of host & $0.000046 \&$ & {$[48,49]$} \\
$\beta_{h_{1}}(t)$ & Transmission rate from mosquitoes to susceptible host & 0.004500 & Periodic \\
$b$ & Bitting rate of mosquitoes & 0.500 & {$[50]$} \\
$\beta_{h_{2}}(t)$ & Transmission rate from mosquitoes to susceptible after & Periodic & {$[26]$} \\
$\beta_{v}(t)$ & losing immunity & & {$[50]$} \\
$v$ & Transmission rate from host to susceptible mosquitoes & Periodic & {$[50]$} \\
$\gamma_{h}$ & Rate of lose of immunity & 0.005 & Assumed \\
$\mu_{v}$ & Recovery rate of host & 0.3288330 & {$[48,49]$} \\
\hline
\end{tabular}



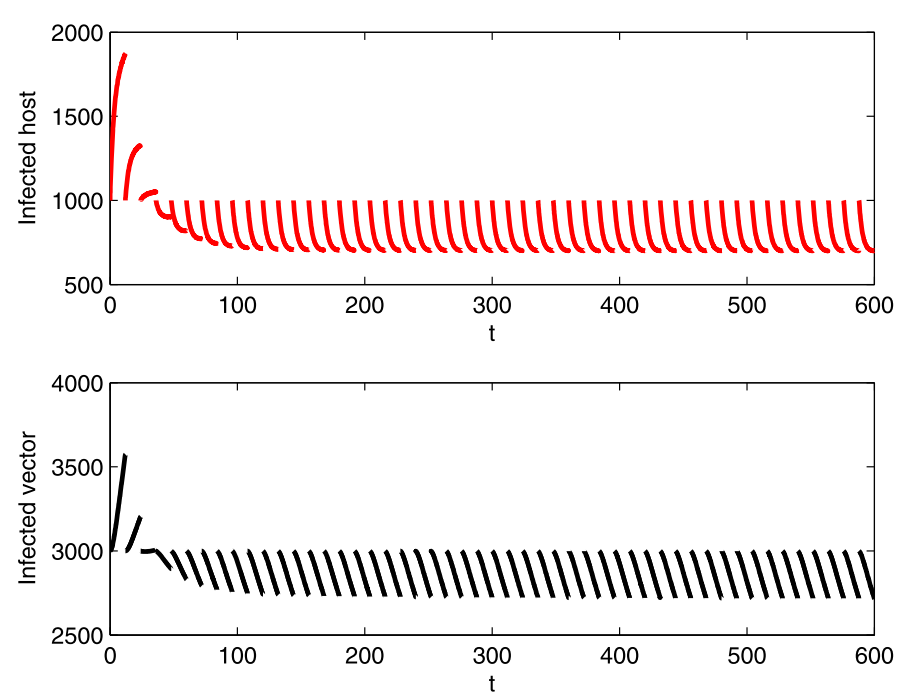

Figure 1 Time series of the infected host individuals $I_{h}$ and infected vector $I_{V}$ under impulsive vaccination strategy, where the red line shows the infected host and the black line shows the infected vector with pulse vaccination strength $q=0.25$
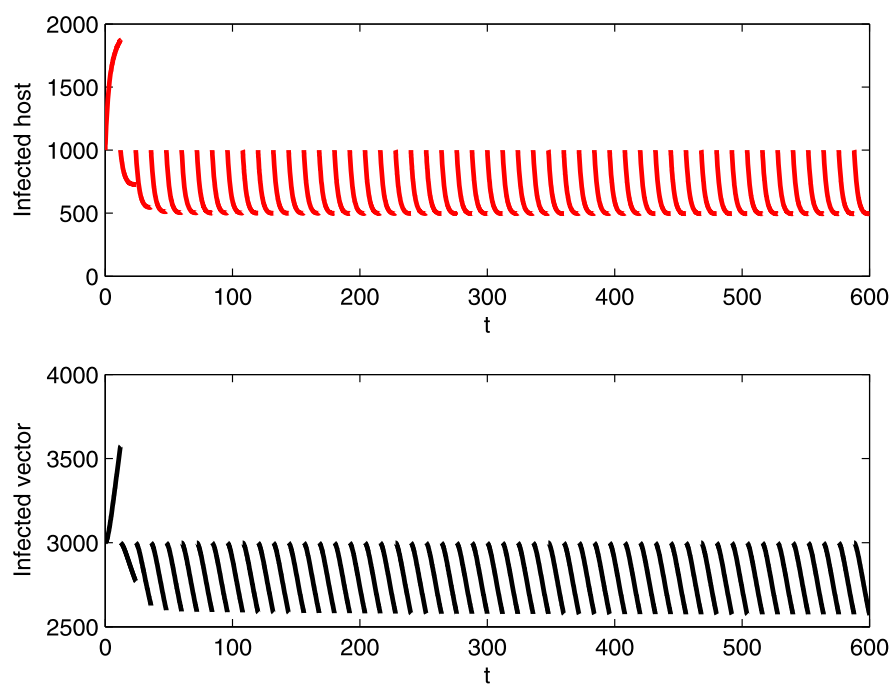

Figure 2 Time series of the infected host individuals $I_{h}$ and infected vector $I_{V}$ under impulsive vaccination strategy, where the red line shows infected host and the black line shows infected vector with pulse vaccination strength $q=0.75$

0.1 to 1 increases the endemic level of the system from 0.26 to 2.34 , and consequently leads to an increase in the size of the outbreak and the level of infection, moreover, $\mathrm{R}_{0}=$ $r\left(\Phi_{(\mathrm{F}-\mathrm{V})(\cdot)}(\mathrm{T})\right)=1$ shows that the critical biting rate is $\bar{b}=0.384$. This indicates that if we control mosquitos such that the biting rate is less than $\bar{b}$, implying that $\mathrm{R}_{0}$ is less than the unity, then the dengue infection can be successfully controlled. We thus emphasize the importance of using mosquito nets which can effectively result in the bitting rate decline. Figure $3(\mathrm{~b})$ shows the variation in the $\mathrm{R}_{0}$ with the variation of the losing rate of immunity $v$, which implies the rate of loss of immunity $v$ does not significantly affect the threshold 


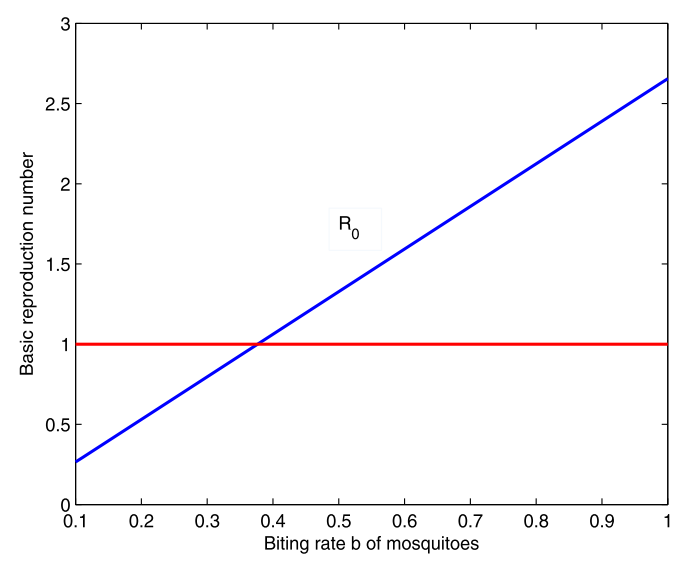

(a)

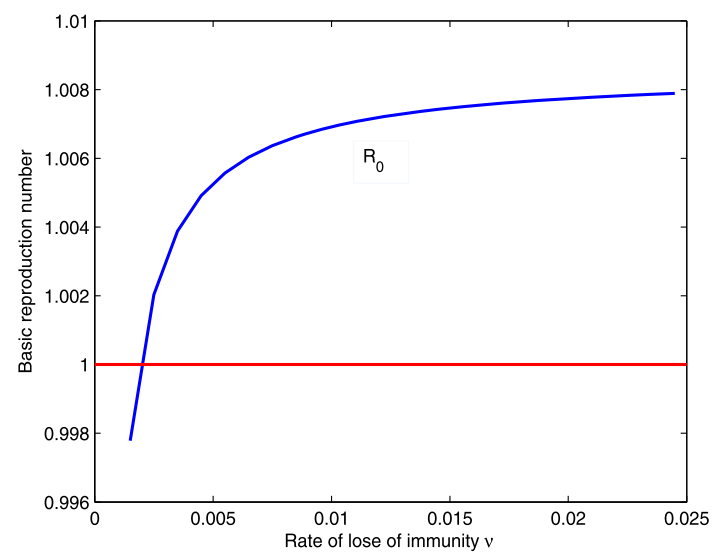

(b)

Figure 3 Variation in the basic reproduction number $R_{0}$ with the mosquitoes biting rate $b(\mathbf{a})$, and with the rate of loss of immunity $v(\mathbf{b})$

parameter $\mathrm{R}_{0}$, indicating that the impact of the partial immunization on lowering the new infection is limited, this happens in dengue infection due to the existence of four serotypes.

To examine the influence of impulsive vaccination on new infections we give the variation in threshold parameter $\mathrm{R}_{0}$ with the intensify $q$ and period of pulse vaccination $T$, shown in Fig. 4(a) and (b). It says that the higher the vaccination rate is, the less the vaccination period is, and the less the basic reproduction number $R_{0}$ is, which implies frequent implementation of the vaccination strategy with great intensity leads to decline of new infections. As more than 50 percent of the world population are in regions with threat of dengue infection and the economic weight of dengue is increasing day by day, pulse vaccination is a beneficial strategy to lower the risk of infection and reduce the economic burden, compared with continuous vaccination strategy in endemic area of dengue fever to control the disease.

\section{Conclusion}

A pulse vaccination policy is an effective measure to prevent infectious diseases, it has contributed successfully in the elimination of several infectious diseases including poliomyelitis and measles. In [51], the advantages of pulse vaccination have been shown 


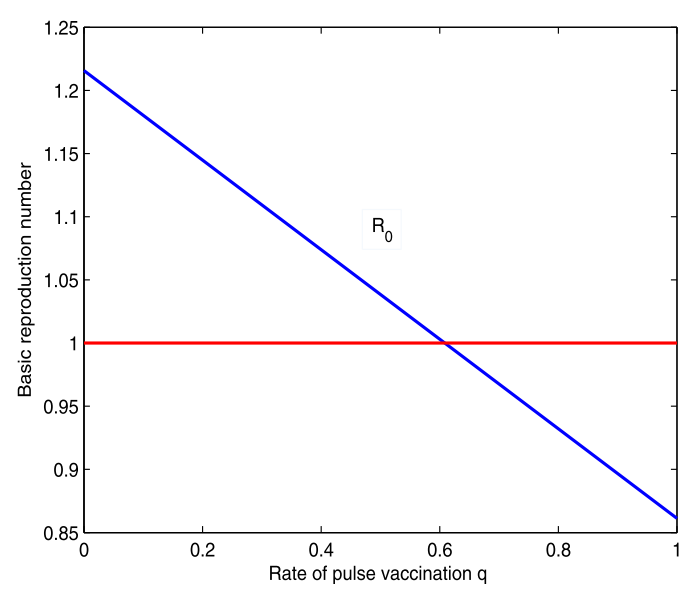

(a)

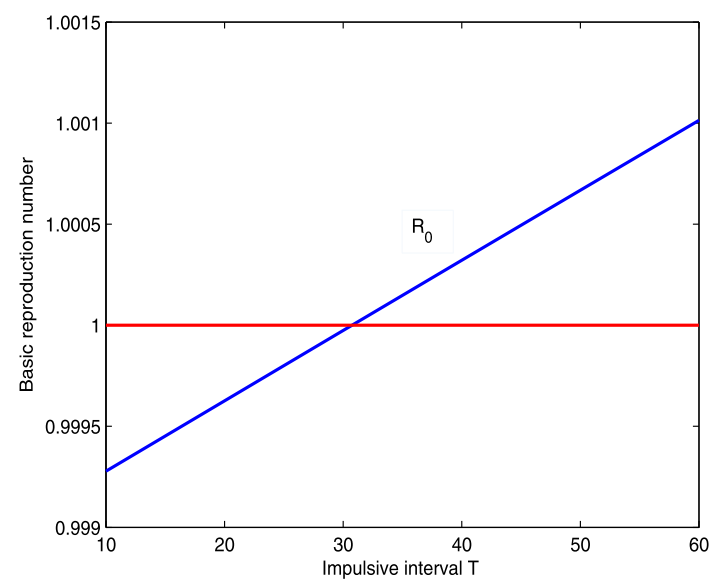

(b)

Figure 4 Variation in the basic reproduction number $R_{0}$ with the vaccination rate $q(\mathbf{a})$, and with the period of vaccination $T(\mathbf{b})$

against measles through mathematical models. Recently, the periodic repetition of vaccination and pulse vaccination policy has attracted the attention of researchers because of the economic and epidemiological factors mentioned in [52-56]. Thus, in this article, we proposed a dynamical model for the transmission pathway of dengue infection with periodic transmission functions and seasonality in the vector population. Moreover, we introduced the strategy of pulse vaccination in the susceptible host population in order to curb the level of dengue infection. We obtained the basic reproduction number, defined as the spectral radius of an infection operator for the proposed impulsive system, which governs whether the disease dies out or not. It has been proven that the disease-free periodic solution is globally asymptotically stable if $R_{0}$ less than unity and is unstable otherwise. We further proved that the dengue infection is uniformly persistent in the community if $R_{0}>1$. Moreover, we numerically demonstrated the influence of pulse vaccination on the infected hosts or infected mosquitos and examined the effect of different input factors on the threshold parameter of the system. The finding indicates that frequent implementation of the vaccination strategy with great intensity leads to a decline of new infections. 


\section{Acknowledgements}

Not applicable.

\section{Funding}

National Natural Sciences Foundation of China, No. 11631012.

\section{Availability of data and materials}

Not applicable.

\section{Competing interests}

We declare that there are no institutional, financial and general competing interests regarding the publication of this research.

Authors' contributions

In this research work, the two authors contributed equally. All authors read and approved the final manuscript.

\section{Publisher's Note}

Springer Nature remains neutral with regard to jurisdictional claims in published maps and institutional affiliations.

\section{Received: 31 May 2019 Accepted: 26 August 2019 Published online: 02 September 2019}

\section{References}

1. Center for Disease Control and Prevention. Dengue, Accessed January 14, 2013. http://www.cdc.gov/dengue/

2. Constenla, D., Armien, B., Arredondo, J., et al.: Costing dengue fever cases and outbreaks: recommendations from a costing dengue working group in the Americas. Value Health Reg. Issues 2015(8), 80-91 (2015)

3. Dengue Signs and Clinical Symptoms. Cbwinfo.com. http://www.cbwinfo.com/Biological/Pathogens/DENV.htm [cited April 23, 2010]

4. Teixeira, M.G., Barreto, M.L.: Diagnosis and management of dengue. Br. Med. J. 339, 4338 (2009)

5. Gubler, D.J.: Dengue and dengue hemorrhagic fever. Clin. Microbiol. Rev. 11(3), 480-496 (1998)

6. Guzman, M.G., Kouri, G., Bravo, J., Valdes, L., Vasquez, S., Halstead, S.B.: Effect of age on outcome of secondary dengue 2 infections. Int. J. Infect. Dis. 6(2), 118-124 (2002)

7. Sabin, A.B.: Research on dengue during World War II. Am. J. Trop. Med. Hyg. 1(1), 30-50 (1952)

8. Siler, J.F., Hall, M.W., Hitchens, A.P.: Dengue: its history, epidemiology, mechanism of transmission, etiology, clinical manifestations, immunity, and prevention. Philipp. J. Sci. 29(20), 1-304 (1952)

9. Rigau-Perez, J.G.: Severe dengue: the need for new case definitions. Lancet Infect. Dis. 6(5), 297-302 (2006)

10. World Health Organisation. Impact of dengue. http://www.who.int/csr/disease/dengue/impact/en/ (2012)

11. Halstead, S.B.: Dengue. Lancet 370, 1644-1652 (2007)

12. Kautner, I., Robinson, M.J., Kuhnle, U.: Dengue virus infection: epidemiology, pathogenesis, clinical presentation, diagnosis, and prevention. J. Pediatr. 131, 516-524 (1997)

13. Rodrigues, H.S., Monteiro, M.T.T., Torres, D.F.M.: Vaccination models and optimal control strategies to dengue. J. Math. Biosci. 247, 1-12 (2014)

14. Fenner, F., Henderson, D.A., Arita, I., Jezek, Z., Ladnyi, I.D.: Smallpox and its eradication. J. Commun. Health 13(4), 245-592 (1988)

15. Hethcote, H.W.: Oscillations in an endemic model for pertussis. Can. Appl. Math. Q. 6(1), 61-88 (1998)

16. Tian, X.H.: Stability analysis of a delayed sirs epidemic model with vaccination and nonlinear incidence. Int. J. Biomath. 5(6), 1250050 (2012)

17. Garba, S.M., Gumel, A.B., Abu Bakar, M.R.: Backward bifurcations in dengue transmission dynamics. Math. Biosci. 215 $11-25(2008)$

18. Pongsumpun, P., Tang, I.M.: Transmission of dengue hemorrhagic fever in an age structured population. Math Comput. Model. 37, 949-961 (2003)

19. Supriatna, A.K., Soewono, E., Van Gils, S.A.: A two-age-classes dengue transmission model. Math. Biosci. 216, 114-121 (2008)

20. De la Sen, M., Agarwal, R.P., Ibeas, A., Alonso-Quesada, S.: On the existence of equilibrium points, boundedness, oscillating behavior and positivity of a sveirs epidemic model under constant and impulsive vaccination. Adv. Differ. Equ. 2011, 748608 (2011)

21. Billings, L., Fiorillo, A., Schwartz, I.B.: Vaccinations in disease models with antibody-dependent enhancement. Math. Biosci. 211, 265-281 (2008)

22. Koopman, J.S., Prevots, D.R., Vaca Marin, M.A., Gomez Dantes, H., Zarate Aquino, M.L., Longini, I.M.J., Sepulveda, A.J. Determinants and predictors of dengue infection in Mexico. Can. Appl. Math. Q. 133(11), 1168-1178 (1991)

23. Esteva, L., Vargas, C.: Analysis of a dengue disease transmission model. Math. Biosci. 150, 131-151 (1998)

24. Esteva, L., Vargas, C.: A model for dengue disease with variable human population. J. Math. Biol. 38(3), $220-240$ (1999)

25. Esteva, L., Vargas, C.: Influence of vertical and mechanical transmission on the dynamics of dengue disease. Math. Biosci. 167(1), 51-64 (2000)

26. Derouich, M., Boutayeb, A., Twizell, E.H.: A model of dengue fever. Biomed. Eng. Online 2, 4 (2003)

27. Syafruddin, S., Noorani, S.M.: Seir model for transmission of dengue fever in Selangor, Malaysia. Int. J. Mod. Phys. Conf. Ser. 9, 380-389 (2012)

28. Feng, Z., Jorge, $X .:$ Velasco-Hernandez, competitive exclusion in a vectorhost model for the dengue fever. J. Math. Biol. 35, 523-544 (1997)

29. Sidarto, K.A.: Mathematical model of dengue disease transmission with severe DHF compartment. Bull. Malays. Math Sci. Soc. 30(2), 143-157 (2007) 
30. Tang, B., Xiao, Y., Tang, S., Wu, J.: Modelling weekly vector control against dengue in the Guangdong Province of China. J. Theor. Biol. 410, 65-76 (2016)

31. Amaku, M., Coutinho, F.A., Raimundo, S.M., Lopez, L.F., Nascimento Burattini, M., Massad, E.: A comparative analysis of the relative eficacy of vector-control strategies against dengue fever. Bull. Math. Biol. 76(3), 697-717 (2014)

32. Tewa, J.J., Dimi, J.L., Bowang, S.: Lypaunov functions for a dengue disease transmission model. Chaos Solitons Fractals (2007). https://doi.org/10.1016/j.chaos.2007.01.069

33. Yang, Y., Xiao, Y.: The effects of population dispersal and pulse vaccination on disease control. Math. Comput. Model. $52,1591-1604(2010)$

34. Zhang, X.B., Xiang, H., Huo, H.F., Meng, X.Y., Xin, Y.: An SIRS epidemic models with pulse vaccinations and non-monotonic incidence rate. Nonlinear Anal. Hybrid Syst. 8, 13-21 (2013)

35. Xiao, Y., Zhao, T., Tang, S.: Dynamics of an infectious diseases with media/psychology induced non-smooth incidence. Math. Biosci. Eng. 10, 455-461 (2013)

36. Song, P., Lou, Y., Xiao, Y.: A spatial SEIRS reaction-diffusion model in heterogeneous environment. J. Differ. Equ. 267(9), 5084-5114 (2019)

37. Zhang, X:: Modelling the effects of augmentation strategies on the control of dengue fever with an impulsive differential equation. Bull. Math. Biol. 78(10), 1968-2010 (2016)

38. De la Sen, M., Agarwal, R.P., Ibeas, A., Alonso-Quesada, S.: On a generalized time-varying seir epidemic model with mixed point and distributed time-varying delays and combined regular and impulsive vaccination controls. Adv. Differ. Equ. 2010, 281612 (2010)

39. Song, X., Jiang, Y., Wei, H.: Analysis of a saturation incidence SVEIRS epidemic model with pulse and two time delays. Appl. Math. Comput. 214(2), 381-390 (2009)

40. Tang, S., Cheke, R.: Stage-dependent impulsive models of integrated pest management (IPM) strategies and their dynamic consequences. J. Math. Biol. 50, 275-292 (2005)

41. Anokhin, A., Berezansky, L., Braverman, E.: Exponential stability of linear delay impulsive differential equations. J. Math Anal. Appl. 193(3), 923-941 (1995)

42. Martinez, M.E.: The calendar of epidemics: seasonal cycles of infectious diseases. PLoS Pathogens 14(11), 1007327 (2018)

43. Cull, P.: Global stability for population models. Bull. Math. Biol. 43, 47-58 (1981)

44. Zhang, F., Zhao, X.Q.: A periodic epidemic model in a patchy environment. J. Math. Anal. Appl. 325, 496-516 (2007)

45. Zhao, X.Q.: Dynamical Systems in Population Biology. Springer, New York (2003)

46. Li, J., Blakeley, D., Smith R.J.: The failure of $r_{0}$. Comput. Math. Methods Med. 13, 224-234 (2011)

47. Smith, H.L., Waltman, P.: The Theory of the Chemostat. Cambridge University Press, Cambridge (1995)

48. KKM.2007. Health Facts (2007)

49. KKRI: dengue fever is still high in South Sulawesi. Tribun Timur Makassar, Newspaper fact (2009)

50. Wang, W., Zhao, X.Q.: Threshold dynamics for compartmental epidemic models in periodic environments. J. Dyn. Differ. Equ. 20(3), 699-717 (2008)

51. Hersh, B.S., Tambini, G., Norgueira, A.C., Carrasco, P., de Quadros, C.A.: Review of regional measles surveilleance in the Americas, 1996-1999. Lancet 355, 1943-1948 (2000)

52. D'Onofrio, A.: Mixed pulse vaccination strategy in epidemic model with realistic distributed infectious and latent times. Appl. Math. Comput. 151, 181-187 (2004)

53. Nokes, D.J., Swinton, J.: The control of a childhood viral infection by pulse vaccination. IMA J. Math. Appl. Med. Biol. $12,29-53(1995)$

54. Nokes, D.J., Swinton, J.: A strategy for global eradication of measles and polio? Trends Microbiol. 5, 14-19 (1997)

55. Shulgin, B., Stone, L., Agur, Z.: Theoretical examinations of pulse vaccination policy in the SIR epidemic model. Math. Comput. Model. 30, 207-215 (2000)

56. Shulgin, B., Stone, L., Agur, Z.: Pulse vaccination strategy in the SIR epidemic model. Bull. Math. Biol. 60, 1123-1148 (1998)

\section{Submit your manuscript to a SpringerOpen ${ }^{\circ}$ journal and benefit from:}

- Convenient online submission

- Rigorous peer review

- Open access: articles freely available online

- High visibility within the field

- Retaining the copyright to your article

Submit your next manuscript at $\gg$ springeropen.com 